\title{
HOLOGRAPHIC INFORMATION MEDIA BASED ON AZO-POLYMERS WITH DIFFERENT STRUCTURES
}

\author{
Irina Davidenko \\ Kiev Taras Shevchenko National University, 64, Volodymyrs'ka str., Kiev 01601, Ukraine \\ email: irynadavydenko@gmail.com
}

\begin{abstract}
Azobenzene polymer compositions are extensively studied now due to wide perspectives of their applications in optoelectronics and information technologies. Some possibilities of modification of their properties by chemical methods are analyzed in the present work. These methods allow to improve information characteristics of the media. Electrooptical effect is investigated and possibilities of polarization sensitive holographic recording is demonstrated in copolymer octyl methacrylate with incorporated azo dyes and its complex with Co ions.
\end{abstract}

Keywords: azobenzene, metallic polycomplexes, polymer composition, electrooptical effect, polarization holography.

\section{Introduction}

Films of polymeric compositions (PC) containing azobenzene dyes or azobenzene lateral groups $[1,2]$ are of interest due to possibility of their use as optically active media [3,4], in particular as electrooptical or magnetooptical light controlling elements and polarization sensitive media for optical holographic recording. Induced polarization appears in the PC films under influence of linearly polarized light which is absorbed by the azobenzene groups and causes changes of isomeric structures. The photoinduced polarization can be conserved at the room temperature during quite a long time. Changes of the polarization are possible under influence of external thermal or mechanical treatments, illumination with light and in external electric or/and magnetic fields. Rotation of the dipole moments happens in an external electric field.

Sensitization of this effect is possible by changing chemical structure of azobenzene groups or by using of different dopants. As it follows from numerous theoretical and experimental investigations $[5,6]$ linearly polarized light is absorbed by the azobenzene groups resulting in changes of isomeric structure. Photoinduced anisotropy of the polarization appears in the films due to changes of concentration of trans- and cis-isomers of the azobenzene groups. Rotation of the dipole moments is possible in an external electric field involving changes of the conditions of interaction between light and PC. This is a fundamental reason of experimentally observed electrooptical effect.

Since the mechanism of external electric influence is determined by the arisen forces orienting the dipole moments of the azobenzene compounds, one can suppose that introduction of metallic ions chemically connected to the polymer could strengthen the electrooptical effect. Also addition of magnetic nanoparticles into the PC could result in external magnetic field influence on photoinduced optical anisotropy [7]. Physical properties of such doped PCs are not completely investigated so far as well as their information properties, i.e. the possibility of registration the diffraction grating, its maximal achievable diffraction efficiency, its control by external electric and magnetic fields.

Azobenzene containing PC are also considered as the perspective media for polarization holography $[6,8]$ due to dependency of the photoinduced optical anisotropy on light polarization. Polarization holography technique $[9,10]$ has many unique properties and possibilities as compared to the scalar holography. These ones result in more flexible opportunities of light beam control when using light polarization sensitive photoactive media.

Investigations of electrooptical properties of PC films, based on azobenzene of different structure depending on presence of coordinated metallic ions as well as on other dopants is the aim of the present work. Holographic information properties of the $\mathrm{PC}$ with and without metallic ions are also studied.

\section{Experimental}

Polymer 4-methacryloyloxy-(4'-carboxy-3'-oxy)azobenzene (A1), its polycomplex with cobalt (A1-Co) and polycomplex 4-methacryloyloxy-(4'carboxy-3'-hydroxy)-2-chlorineazobenzene with cobalt ( $\mathrm{A} 1-\mathrm{Co}, \mathrm{Cl})$ were synthesized and investigated (Figure 1). Their characterization accordingly to chemical standards and the full scheme of synthesis is presented by authors [11]. Structural formulas of the polycomplexes A1, A1-Co and A1-Co,Cl are shown below.

There is inter-chain coordination of metallic ions in the polycomplexes A1-Co. The polycomplex A1-Co,Cl contains chlorine increasing dipole moment of the azobenzene fragment. The samples were prepared as the structures with free surface of the polymeric film: glass substrate - polymeric film. The thickness of the polymeric film was $1.8-2.0 \mu \mathrm{m}$. 
<smiles>CC(C)(CI)C(=O)Oc1ccc(N=Nc2ccc(C(=O)O)c(O)c2)cc1</smiles>

Al

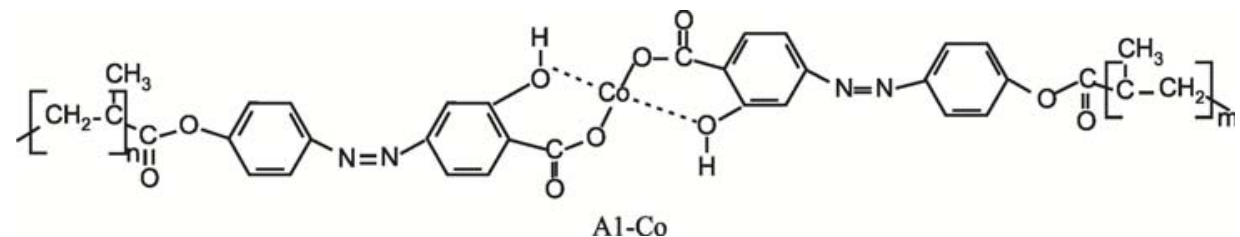

Al-Co

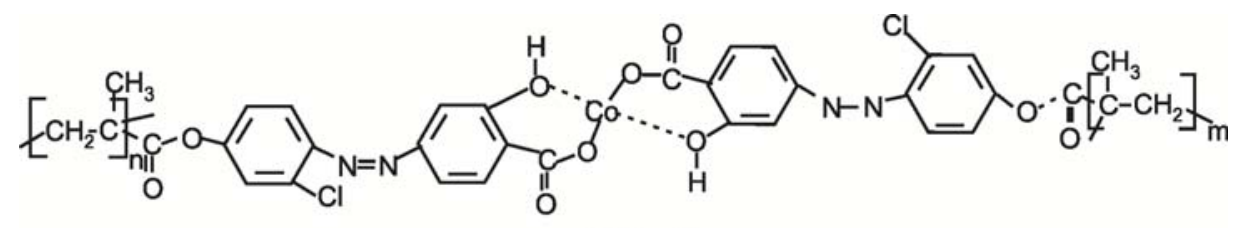

$\mathrm{A} 1-\mathrm{Co}, \mathrm{Cl}$

Figure 1. Polymer 4-methacryloyloxy-(4'-carboxy-3'-oxy)azobenzene (A1), its polycomplex with cobalt (A1-Co) and polycomplex 4-methacryloyloxy-(4'carboxy-3'-hydroxy)-2-chlorineazobenzene with cobalt $(\mathrm{A} 1-\mathrm{Co}, \mathrm{Cl})$.

Results of investigation of an influence of external electric field on the transmission of the films A1 and A1-Co are presented in Figure 2. Here, $I_{0}$ and $I_{E}$ are the intensities of light before and after application of the external electric field respectively. External electric field $E=1 \times 10^{8} \mathrm{~V} / \mathrm{m}$ in the PC film was produced by a crown discharge in a special device. The field was oriented normally to the film surface. It was observed [12] that influence of electric field increases when the samples were previously illuminated with linearly polarized light from the band of absorption of azobenzene groups $(\lambda<550 \mathrm{~nm})$. The most pronounced changes of $I_{0}$ when switching on external electric field were observed for the time of preliminary illumination $\mathrm{t}>30 \mathrm{~s}$. External electric field involves decrease of the intensity of light passed through the samples with compound A1 (Figure 2, curve 1) and its growth for the samples with A1-Co (Figure 2, curve 2). All measurements were done when the investigated samples were placed between crossed polarizers.

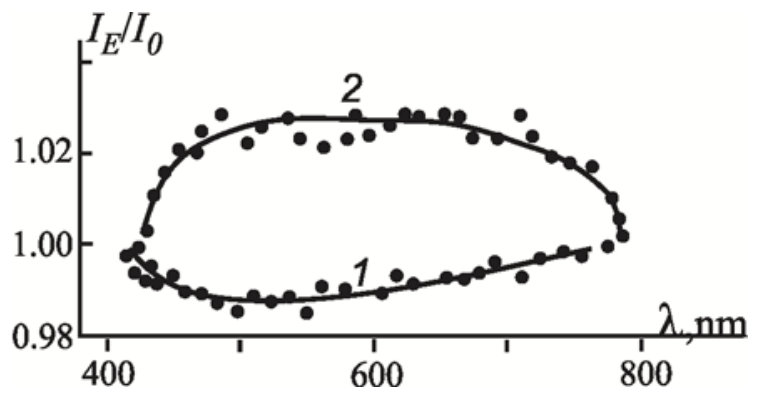

Figure 2. Spectral dependencies of $I_{E} / I_{0}$ in the samples with A1 (1), A1-Co (2).

Recording of the polarization holographic gratings in the samples with the polycomplex $\mathrm{A} 1-\mathrm{Co}, \mathrm{Cl}$ can be considered as an example. $\mathrm{Nd}^{3+}$ : YAG laser with light wavelength $\lambda=532 \mathrm{~nm}$ and maximal power $100 \mathrm{~mW}$ was used. Usual setup for registration of the holograms of flat wave front $[9,13]$ was used in the experiments. Kinetics of recording and relaxation of the holographic gratings for the both cases mutually parallel and perpendicular polarization vectors of recording and reference light beams are shown in Figure 3. The ratio between intensities of the beams was 1:1. The diffraction efficiency $\eta$ was determined as the ratio between light intensity in -1 diffraction order and intensity of the reference beam passed through the sample. The spatial frequency of the hologram of flat wave front was $1500 \mathrm{~mm}^{-1}$. 

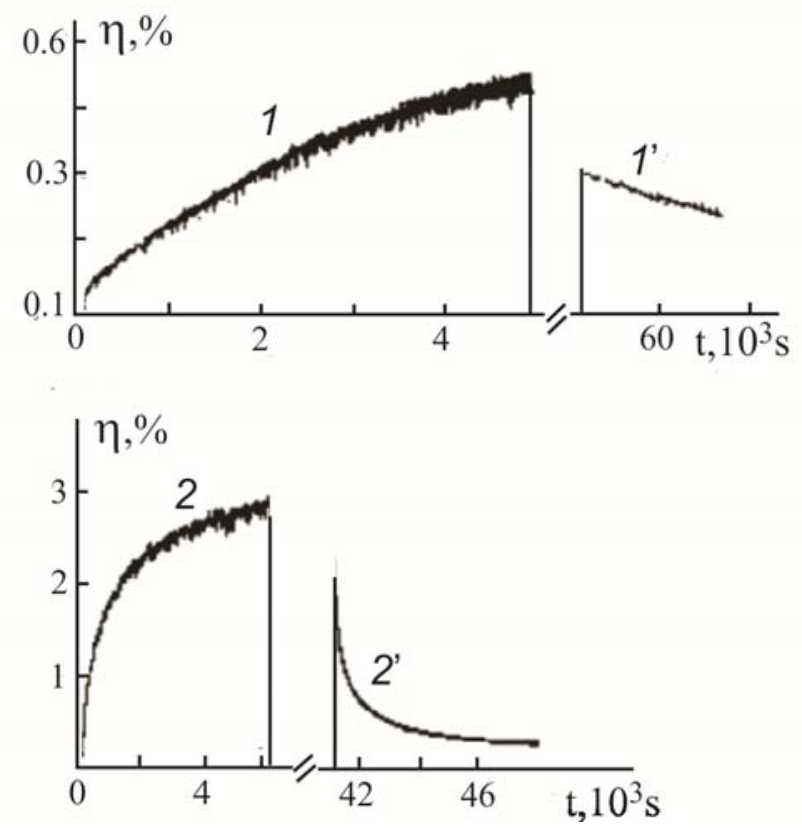

Figure 3. Kinetics of recording $(1,2)$ and relaxation $\left(1 ', 2\right.$ ') of the holograms for the cases $\vec{e}_{1} \| \vec{e}_{2}\left(1,1^{\prime}\right)$ and $\vec{e}_{1} \perp \vec{e}_{2}\left(2,2^{\prime}\right)$ in the sample with A1-Co,Cl. The curves 1', 2' were registered using reference beam after exposure and keeping of the recording medium in darkness.

The case when light polarization vectors $\vec{e}_{1}$ and $\vec{e}_{2}$ of the object and reference beams are parallel $\vec{\theta}_{1} \| \vec{\theta}_{2}$ corresponds to spatial distribution of the light intensity along the sample surface. When these vectors are mutually perpendicular spatial distribution of ellipticity and orientation of polarization ellipse of the total light beam along the sample surface takes place. After registration of the holograms their relaxation occurs, and its velocity increases when the samples is illuminated with continuous light. For $\vec{e}_{1} \| \vec{e}_{2}$ diffraction efficiency $\eta$ is less and relaxation time is greater as compare to $\vec{e}_{1} \perp \vec{e}_{2}$. For the first case recorded holograms can be conserved at the room temperature during few months.

Polycomplex of azobenzene with intrachain coordination of Co ion (A2-Co) was synthesized and investigated to compare electrooptical properties of this one and polycomplex of azobenzene with interchain coordination of Co ion (A1-Co) (Figure 4).

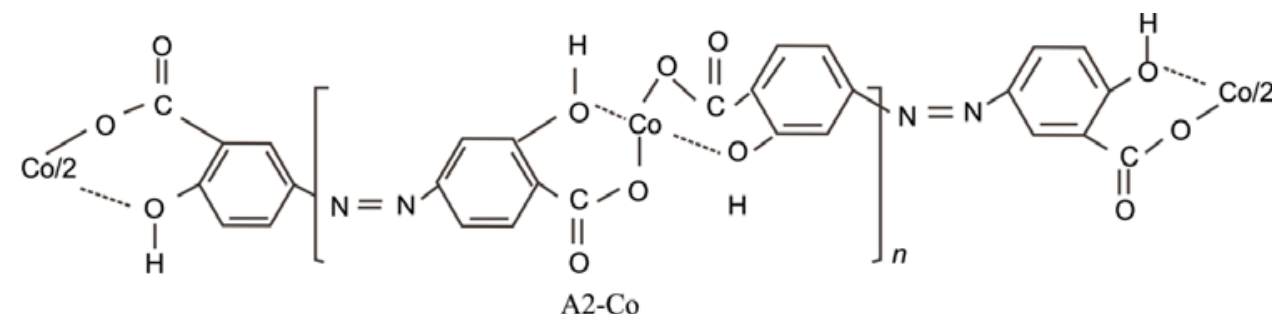

Figure 4. Polycomplex 4- carboxy-3-oxy-3'-carboxy-4'oxyazobenzene with cobalt.

Spectral dependencies of $\delta I_{E}$ in the samples with PC A1-Co (curve 1) and A2-Co (curve 2) are shown in Figure 5. Here, $\delta I_{E}=\left(I_{E}-I_{0}\right) / I_{0}$ where $I_{E}$ and $I_{0}$ are the intensities of light before and after application of the external electric field respectively. Measurements were done for crossed polarizers. The samples were preliminary illuminated with linearly polarized light from the band of absorption of azobenzene groups $\lambda<550 \mathrm{~nm}$. 


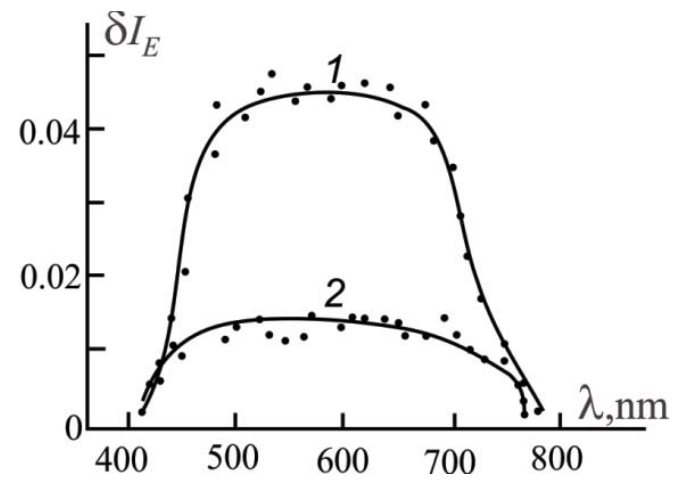

Figure 5. Spectral dependencies of $\delta I_{E}$ in the films with A1-Co (1) and A2-Co (2) for crossed polarizers after preliminary illumination with linearly polarized light $\lambda<550 \mathrm{~nm}$ during $60 \mathrm{~min}$.

\section{Results and discussion}

Opposite sign of influence of external electric field on the intensity of light passed through the samples with PC A1 and A1-Co can be explained by the peculiarities of interaction of these compounds with external electric field. In the films A1 an external electric field provokes alignment of the photoinduced dipoles along the force lines of the field. As a result, interaction between polarized light and these dipoles is weaker and $\delta I E<0$ for crossed polarizers. In the films A1-Co in the external electric field polarized light undergoes more intensive dispersion and depolarization resulting in positive $\delta I E$. This fact is probably caused by more effective interaction between the $\mathrm{Co}^{2+}$ ions and the electric field as compared to dipoles interaction with the field. These ions are bonded to the azobenzene groups indirectly, and the direction of the bond and the photoinduced dipole moment do not coincide. Hence, change of orientation of the photoinduced dipoles bonded to the metallic ions in the sample with A1-Co in the external electric field could be opposite to the change in the sample with A1. These experimental results can be qualitatively described within the scope of phenomenological theoretical model [12].

The samples with polycomplex A1-Co, $\mathrm{Cl}$ was chosen as an example for holographic recording. It is once more chemical modification of investigated azobenzene polycomplexes with cobalt ions. It is characterized by increased dipole moment of azobenzene fragment due to presence of chlorine ions. Recording of two types of interference field of the recording and reference beams was accomplished: spatial distribution of light intensity $\left(\vec{e}_{1} \| \vec{e}_{2}\right)$ and spatial distribution of light polarization state $\left(\vec{e}_{1} \perp \vec{e}_{2}\right)$. Difference of the relaxation times (Figure 3 ) in the both cases can be attributed to the difference of the structural rearrangement of the polymeric matrix which happens in the polymeric film containing azobenzene polycomplexes while intensive trans-cis-izomerization of the azobenzene groups occurred. Similar effects were observed before $[14,15]$ and it was concluded that exactly rearrangement of the polymeric matrix is the reason of long time conservation of the holographic recording. Moreover, long-lived photoinduced surface relief $[6,16]$ can contribute in the observed effect.

Co ions coordinated by chelate groups (A2-Co) participate in electrooptical effect and create additional mechanical obstacles for izomerization of azobenzene groups and their rotation in the external electric field. Therefore, we observed weaker electrooptical effect in the films with A2-Co where azobenzene group is bonded to two Co ions (Figure 5).

\section{Conclusions}

Azobenzene polymers now are widely considered as the perspective media for information technologies. Their physical and information properties are extensively studied. Analyzing few experimental results presented in this paper one can conclude that these properties can be varied depending on practical necessities by appropriate chemical modifications, namely by introduction of coordinated metallic ions, by changes of the dipole moment of azobenzene fragments, by change of coordination of the metallic ions. Introduction of coordinated metallic ions in the investigated PC opens additional possibilities of control of the properties of the photoactive medium, allows increasing of their sensitivity in external fields. Special conditions can be chosen when presence of metallic ions and/or change of dipole moment of azobenzene groups intensify the effect of photoinduced polarization.

Moreover, physical and information properties of PC containing azobenzene compounds can be changed by introduction of organic merocyanine dyes into their structure [17]. Their influence in the PC reveals itself in sensitization of electrooptical effect. Also increase of the length of "spacers" and possibility to form hydrogen bonds between substitutes in azobenzene groups reduce time and increase magnitude of the changes of optical characteristics of the films under influence of external electric field [18]. Since influence of external electric field is explained by appearance of the forces effecting on the dipole moments of azobenzene groups and metallic ions increase of the dipole moments of azobenzene groups by chemical methods provokes decrease of the metallic ions influence on the electrooptical properties. This effect was observed by authors [19] introducing donor and acceptor substitutes into the structure of azobenzene groups. 
Thus, polymer composites with azobenzene dyes and their metallic complexes have wide perspectives for practical applications such as for holographic information recording and in optoelectronics due to possibilities to vary their properties particularly by chemical methods. Some of these methods are analyzed in this paper.

\section{References}

1. Yesodha, S.K.; Sadashiva, C.K.; Pillai, P.; Tsutsumi, N. Stable polymeric materials for nonlinear optics: a review based on azobenzene systems. Progress in Polymer Science, 2004, 29, pp. 45-74.

2. Yaroshchuk, O.; Tereshcenko, A.; Zakrevskyy, Yu.; Shanski, I. 3D Orientational Structures in Azopolymers Studied by UV Absorption Method. Molecular Crystals and Liquid Crystals, 2001, 361, pp. 187-192.

3. Camorani, P.; Cristofolini, L.; Fontana, M.P.; Angiolini, L. Comparative Study of Photomechanical Effects in Linear and Star Azo-Polymers. Molecular Crystals and Liquid Crystals, 2009, 500, pp. 1-9.

4. Ishitobi, H.; Tanbe, M. The anisotropic nanomovement of azo-polymers. Optics Express, 2007, 15, pp. 652-659.

5. Lefin, P.; Fiorini, C.; Nunziy, J.-M. Anisotropy of the photo-induced translation diffusion of azobenzene dyes in polymer matrices. Pure Appl. Opt., 1998, 7, pp. 71-82.

6. Bublitz, D.; Fleck, B.; Wenke, L. A model for surface-relief formation in azobenzene polymers. Applied Physics B, 2001, 72, pp. 931-936.

7. Borsch, A.A.; Volkov, V.I.; Lyakhovetskii, V.R.; Kutsenko, A.S. Nonlinear refraction in an epoxy-based polymer with 4-aminoazobenzene caused by the orientation of azobenzene molecules upon pulsed laser excitation. Quantum Electronics, 2003, 33, pp. 441-445.

8. Eickman, J.; Bieringer, T.; Kostromine, S.; Berneth, H.; Thoma, R. Photoaddressable Polymers: A New Class of Materials for Optical Data Storage and Holographic Memories. Japanese Journal of Applied Physics, 1999, 38, pp. 1835-1840.

9. Todorov, T.; Nikolova, L.; Tomova, N. Polarization holography. 1: A new high-efficiency organic material with reversible photoinduced birefringence. Applied Optics, 1984, 23, pp. 4309-4312.

10. Yang, Q.; Wei, Z.; Zhang, Y.; Sun, G.; Li, F. Polarized-light-controlled holographic recording in an azobenzenedoped polymer film. Applied Physics B, 2001, 72, pp. 855-858.

11. Davidenko, N.A.; Davidenko, I.I.; Pavlov, V.A.; Popenaka, A.N.; Savchenko, I.A.; Shumeluk, A.N. Recording medium based on a polymer azobenzene complex with cobalt for polarization holography. Theoretical and Experimental Chemistry, 2009, 45, pp. 54-57.

12. Davidenko, N.A.; Davidenko, I.I.; Savchenko, I.A.; Popenaka, A.N.; Baath, L.B. Electrooptical effect in films of azobenzene polycomplexes with cobalt. Journal of Applied Physics, 2008, 103, pp.094223-094227.

13. Caulfield, H.J. Handbook of Optical Holography; Academic Press: New-York, 1979, 480 p.

14. Freiberg, S.; Lagugne-Labarthet, F.; Rochon, P.; Natansohn A. Synthesis and Characterization of a Series of Azobenzene-Containing Side-Chain Liquid Crystalline Polymers. Macromolecules, 2003, 36, pp. 2680-2688.

15. Davidenko, I.I.; Savchenko, I.A.; Popenaka, A.N.; Shumelyuk, A.N.; Bedarev, V.A. Holographic recording and electrooptical effect in the films of azobenzene polycomplexes with cobalt. Ferroelectrics, 2007, 352, pp. 158-163.

16. Viswanathan, N.K.; Balasubramanian, S.; Li, L.; Sukant, K.; Tripathy, K.; Kumar, J. A Detailed Investigation of the Polarization-Dependent Surface-Relief-Grating Formation Process on Azo Polymer Films. Japanese Journal of Applied Physics, 1999, 38, pp. 5928-5937.

17. Davidenko, N.A.; Davidenko, I.I.; Savchenko, I.A.; Popenaka, A.N.; Derevyanko, N.A.; Ishchenko, A.A.; Kulinich, A.V. Sensitization of the Electrooptical Effect by a Merocyanine Dye in Polymers of Azobenzene Derivatives. High Energy Chemistry, 2008, 42, pp. 123-126.

18. Davidenko, N.A.; Davidenko, I.I.; Popenaka, A.N.; Savchenko, I.A.; Studzinskii, S.L. Effect of the interaction of azobenzene groups in polymeric composites on their electrooptical characteristics. Theoretical and Experimetal Chemistry, 2009, 45, pp. 349-354.

19. Davidenko, N.A.; Davidenko, I.I.; Popenaka, A.N.; Savchenko, I.A. Electrooptical properties of the films of azopolymers and polycomplexes with donor and acceptor substitutes. Optical Journal, 2008, 75, pp. $70-73$ (In Russian). 